\title{
Evaluation of the Efficacy of Potassium lodide Preparation for Radioactive lodine Therapy in Graves' Disease: A Retrospective Chart Review
}

\author{
Seigo Tachibana ${ }^{\mathrm{a}, \mathrm{b}}$, , Hiroyuki Yamashita ${ }^{\mathrm{c}}$, Toshihiko Yanase ${ }^{\mathrm{b}}$
}

\begin{abstract}
Background: Pretreatment by antithyroid drugs (ATDs) before radioactive iodine therapy (RAIT) for Graves' disease (GD) is often performed to avoid aggravation of thyroid function including thyroid storm. However, in patients suffering from adverse effects of ATDs, potassium iodide (KI) is often selected to treat hyperthyroidism before RAIT in Japan. The aim of this study was to evaluate the efficacy of KI preparation for RAIT in GD and whether a short-term discontinuation of KI before RAIT is a safe and useful method in GD patients showing adverse effects from ATDs.

Methods: This is a retrospective chart review. The 24 patients enrolled in this study were divided into two groups. Patients who had large goiter and/or underwent previous RAIT were excluded. Before RAIT, 11 patients were administered KI and were referred to as the KI group. The remaining 13 patients were administered methimazole (MMI) and referred to as the MMI group. Between these two groups, the following factors were compared; radioactive iodine uptake (RAIU) before RAIT, thyroid gland weight, dose of administered 131-I, dose of 131-I based on thyroid gland weight, free thyroxine (FT4) at RAIT, difference in FT4 between before and 4 days after discontinuation of drugs, and thyroid function and thyroid gland weight 1 year after RAIT.
\end{abstract}

Results: RAIU before RAIT ( $\mathrm{P}=0.0018)$, dose of 131-I $(\mathrm{P}=0.0037)$, FT4 at RAIT $(\mathrm{P}=0.0034)$, and thyroid gland weight 1 year after RAIT $(P=0.0065)$ showed significant differences. Thyroid gland weight, dose of 131-I based on thyroid gland weight, difference in FT4 between before drug discontinuation and at RAIT, and thyroid function at 1 year after RAIT did not show any significant differences.

Manuscript accepted for publication January 03, 2017

aDepartment of Endocrinology, Yamashita Thyroid and Parathyroid Clinic, 1-8 Shimogofukumachi, Hakata-ku, Fukuoka City 812-0034, Japan

bDepartment of Endocrinology and Diabetes Medicine, Fukuoka University Hospital, 7-45-1 Nanakuma, Jonan-ku, Fukuoka City 814-0180, Japan

'Department of Surgery, Yamashita Thyroid and Parathyroid Clinic, 1-8 Shimogofukumachi, Hakata-ku, Fukuoka City 812-0034, Japan

${ }^{\mathrm{d} C}$ Corresponding Author: Seigo Tachibana, Department of Endocrinology, Yamashita Thyroid and Parathyroid Clinic, 1-8 Shimogofukumachi, Hakataku, Fukuoka City 812-0034, Japan. Email: Tachibana@kojosen.com

doi: https://doi.org/10.14740/jem394w
Conclusion: These results suggest that most patients without large goiter and/or highly aggressive disease may be safely and efficiently treated with RAIT and KI pretreatment until 4 days before therapy.

Keywords: Graves' disease; Radioactive iodine therapy; Potassium iodide; Antithyroid drug

\section{Introduction}

Radioactive iodine therapy (RAIT) is one effective treatment for Graves' disease (GD). RAIT for GD was first introduced approximately 70 years ago, and the utility of this procedure is well known [1]. In Japan, the first choice for GD treatment is an antithyroid drug (ATD); however, when patients show adverse ATD effects, and/or GD does not go into remission with ATDs, RAIT and thyroidectomy are the second treatment options [2]. Endocrinologists try to treat hyperthyroidism by ATDs and/or potassium iodide (KI) before RAIT to prevent thyroid storm after RAIT. Burch et al reported that among 709 survey respondents who were endocrinologists, $352(49.6 \%)$ used ATD preparations only in selected patients and $37.7 \%$ did routinely in most patients [3]. Their report indicated that not only iodine restriction before RAIT, but also discontinuation of ATDs is often selected in numerous cases. As well as cases administered ATDs, when a patient is taking KI for the treatment of adverse ATD effects, KI should be discontinued before RAIT. However, discontinuation of ATD and/or KI is one of the risk factors of thyroid function aggravation accompanying RAIT. In order to avoid this risk, Zakavi et al evaluated methimazole (MMI) discontinuation before RAIT and reported that a short duration of ATD withdrawal before RAIT is safe and useful [4]. In fact, in the above Burch survey, $37.1 \%$ of the responders stopped ATDs at 7 days, $25.2 \%$ at 5 days, $12.1 \%$ at 4 days, and $15.8 \%$ at 3 days before RAIT [3]. On the other hand, very few studies have focused on a short duration of KI withdrawal before RAIT. In several countries, KI administration for GD is not recommended except in preparation for GD surgery, treatment for thyroid storm, or treatment after RAIT [5-11]. However, in Japan, a high-iodine-intake area, $\mathrm{KI}$ is reported as a useful drug for GD treatment. Hirakawa et al reported that restriction of dietary iodine does not ameliorate the early effects of ATDs in an area of excessive iodine intake [12]. Takata et al demonstrated 
Table 1. Clinical Features of $\mathrm{KI}$ and MMI Groups

\begin{tabular}{|c|c|c|c|}
\hline & KI & MMI & P-value \\
\hline Age $($ mean $\pm \mathrm{SD})$ & $41.9 \pm 11.4$ & $39.2 \pm 13.6$ & n.s. \\
\hline Gender (male/female) & $2: 9$ & $0: 13$ & n.s. \\
\hline Smoking (yes/no) & $3: 8$ & $2: 11$ & n.s. \\
\hline Duration of GD treatment (median $(25-75 \%)$ ), months & $16.0(3.0-52.0)$ & $37.0(16.5-58.5)$ & n.s. \\
\hline FT4 before discontinuation of KI or MMI (median $(25-75 \%)$ ), ng/dL & $2.11(1.58-2.48)$ & $1.21(1.00-1.49)$ & $\mathrm{P}<0.05$ \\
\hline Method of RAIT (fixed dose/calculated dose) & $8: 3$ & $3: 10$ & $\mathrm{P}<0.05$ \\
\hline Dose of drugs before RAIT, (median (25-75\%)), mg & & & - \\
\hline MMI (n = 0:13) & - & $12.5(7.5-16.3)$ & \\
\hline $\mathrm{KI}(\mathrm{n}=11: 0)$ & $150(100-200)$ & - & \\
\hline
\end{tabular}

the usefulness of KI combined with ATD therapy [13]. In addition, Okamura et al reported the effectiveness of only KI in GD patients who showed adverse effects to MMI [14]. Therefore, in Japan, KI is often administered to GD patients who are showing adverse ATD effects. In addition, when GD patients showing adverse ATD effects are treated by RAIT, KI is often selected to avoid aggravation of thyroid function.

\section{Materials and Methods}

\section{Objective}

In this study, we evaluated preparation for RAIT by KI, and whether a short duration of KI withdrawal before RAIT is a safe and useful method in GD patients.

\section{Subjects}

RAIT was performed in 56 GD patients from May 2011 to October 2014 in our institution. Of the 56 patients, 24 were enrolled in this study. Thirty-two patients were excluded, because they dropped out after RAIT or RAIT was not performed in accordance with the following protocol and/or exclusion criteria. The exclusion criteria were defined as follows: large goiter and/ or experience of previous RAIT. The definition of large goiter in exclusion criteria was determined that estimated thyroid gland weight was more than $50 \mathrm{~g}$. In Japan, a single administered dose of 131-I RAIT for outpatients is restricted to within $13 \mathrm{mCi}$. If patients have large goiter, it is difficult to evaluate the efficacy of single RAIT because an enough high dose of 131-I to treat hyperthyroidism cannot be administered. Therefore, in this study, we determined the above exclusion criteria of thyroid gland weight. All enrolled patients were diagnosed with GD by thyrotoxicosis with positive thyroid stimulating hormone receptor antibody (TRAb) and/or high radioactive iodine uptake (RAIU). Neck ultrasonography was performed in all patients to exclude patients with an autonomously functioning thyroid nodule and possible malignant tumor. All patients were treated with RAIT for the following reasons. Of the 24 pa- tients, 10 showed adverse effects from ATDs: four showed liver dysfunction, four showed drug eruption, one showed arthralgia, one showed drug fever, and two showed agranulocytosis. Some patients experienced multiple adverse effects at the same time. Thirteen were unable to go into remission with ATDs, and one chose RAIT. For the preparation of RAIT, 11 patients were administered KI, and 13 were administered MMI. The dose of drugs before RAIT is shown in Table 1.

\section{Protocol of RAIT}

Before RAIU for RAIT, oral-iodine-intake was restricted for 7 days in patients treated with only ATDs, and for 4 days in those treated with KI. MMI and KI were withdrawn 4 days before RAIU for RAIT. Duration of restriction of oral-iodine-intake and discontinuation of MMI or KI was adopted according to the guidelines for RAIT from Japan Thyroid Association (JTA) in 2007 [15]. RAIU was evaluated $3 \mathrm{~h}$ after intake of $131-\mathrm{I}$ at a dose of $0.1 \mathrm{mCi}$. The dose of administered $131-\mathrm{I}$ was $13.0 \mathrm{mCi}$ as the fixed dose or the calculated dose. The determination of the fixed dose or the calculated dose of 131-I was by doctor's decision in consideration of the patient clinical background. For example, if the patient had relatively large goiter and/or could not take ATDs because of adverse effects, the fixed dose method was often selected, because immediate treatment of hyperthyroidism was preferred. The calculated dose was determined by the following formula [16]: calculated dose $(\mathrm{mCi})=$ (thyroid gland weight $(\mathrm{g})) \times(80-160 \mu \mathrm{Ci} 131-\mathrm{I} / \mathrm{g}$ of thyroid)/ $($ RAIU $(\%)) \times 10$. The dose of 131-I based on thyroid gland weight was determined between 80 and $160 \mu \mathrm{Ci} / \mathrm{g}$. If a euthyroid state was expected after RAIT, the dose of 131-I based on thyroid gland weight was determined between 80 and 120 $\mu \mathrm{Ci} / \mathrm{g}$. However, if hypothyroidism was acceptable after RAIT, it was determined between 140 and $160 \mu \mathrm{Ci} / \mathrm{g}$. Thyroid gland weight was estimated by thyroid US. After RAIT, discontinuation of MMI or KI, and iodine restriction continued for 3 days.

\section{Methods}

This was a retrospective chart review. The 24 enrolled patients 


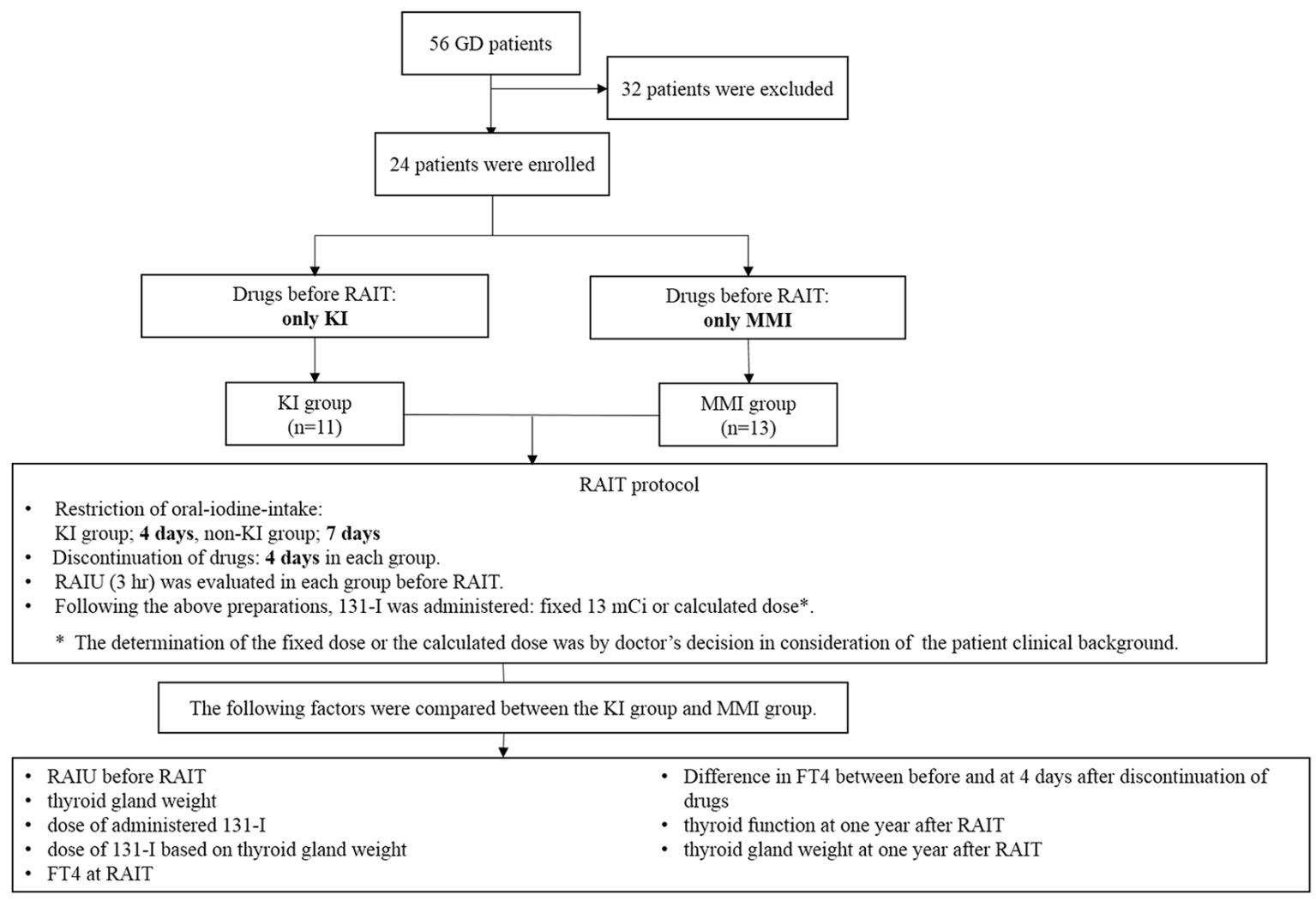

Figure 1. The protocol of this study.

were divided into the following two groups. Before RAIT, KI was administered to 11 patients (two male and nine female) who were referred to as the KI group, and MMI was administered to 13 female patients who were referred to as the MMI group. The clinical features of these two groups are shown in Table 1.

Between these two groups, we compared the following factors: RAIU before RAIT, thyroid gland weight, dose of administered 131-I, dose of 131-I based on thyroid gland weight, free thyroxine (FT4) at RAIT, variation of FT4 between before and at 4 days after discontinuation of drugs, thyroid function at 1 year after RAIT and thyroid gland weight at 1 year after RAIT. The thyroid function at 1 year after RAIT was evaluated on the basis of whether each subject was taking MMI or KI 1 year after RAIT or not. Patients who were taking MMI or KI were defined as non-remissive (non-R), and patients who were not taking both MMI and KI were defined as in remission (R). The protocol of this study is shown in Figure 1.

\section{Laboratory measurements}

Serum levels of thyroid stimulating hormone (TSH), FT4 and TRAb were determined using an ECLusys kit (Roche Diagnostics, Penzberg, Germany). The reference ranges were as follows: TSH 0.5 - $5.0 \mathrm{mIU} / \mathrm{L}$ and FT4 $0.9-1.7 \mathrm{ng} / \mathrm{dL}$. TRAb measurement was determined with the anti-M22 antibody, third generation. The reference range of TRAb was below 2.0
$\mathrm{IU} / \mathrm{L}$.

\section{Ethics approval}

Approval to undertake the study was received from the Research Ethics Committee in Yamashita Thyroid and Parathyroid Clinic (2016-2).

\section{Statistical analysis}

In these two groups, statistical analysis was performed with Fisher's exact test and the Mann-Whitney U test using JMP ver. 11.0 (SAS Institute Inc.).

\section{Results}

There were significant statistical differences in RAIU before RAIT, dose of 131-I, FT4 at RAIT, and thyroid gland weight at 1 year after RAIT between the KI group and MMI group (Table 2). There were no significant differences in thyroid gland weight, dose of 131-I based on thyroid gland weight, difference in FT4 between before and 4 days after discontinuation of drugs, and thyroid function at 1 year after RAIT between the KI group and MMI group (Table 2). RAIU of the KI group was lower than that of the MMI group, approximately half of the 
Table 2. Results of This Study

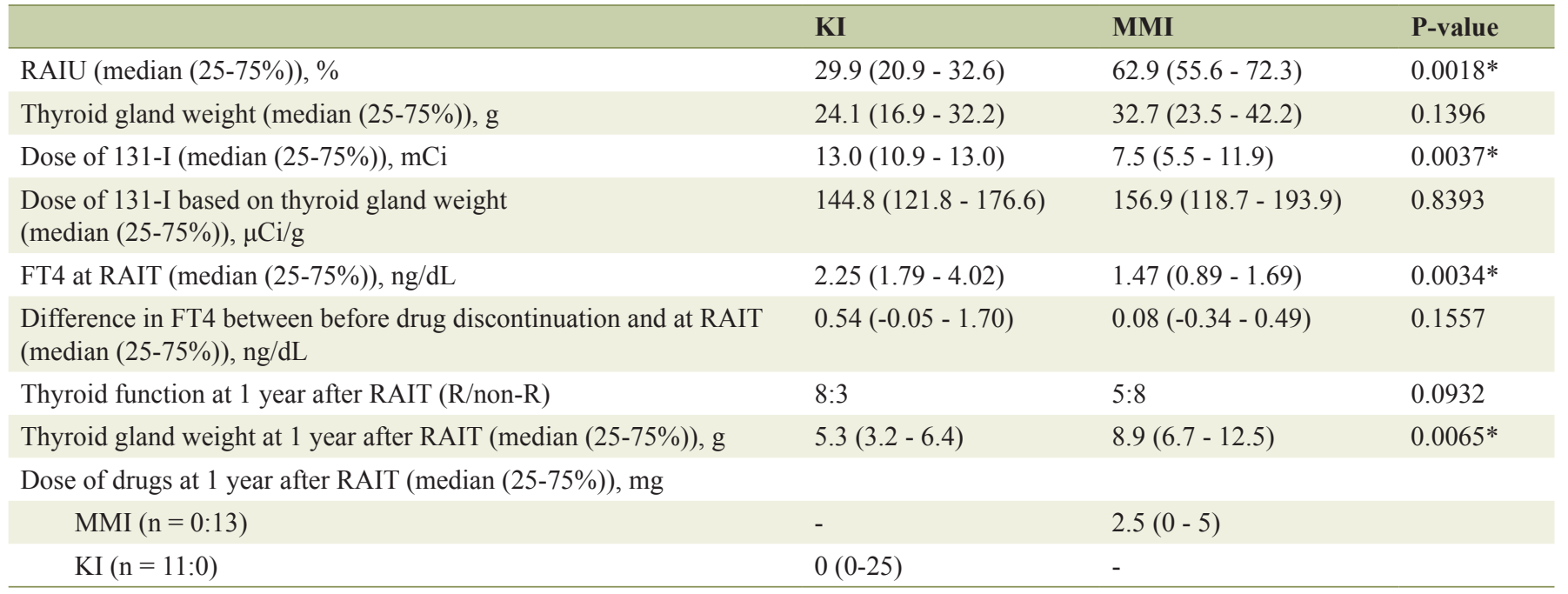

RAIU of the MMI group was equivalent to that of the KI group $(\mathrm{P}=0.0018)$. The 131-I administration dose of the KI group was higher than that of the MMI group $(\mathrm{P}=0.0037)$. Thyroid gland weight and dose of 131-I based on thyroid gland weight of the KI group was equivalent to the MMI group $(\mathrm{P}=0.1396$, $\mathrm{P}=0.8393)$. FT4 at RAIT in the KI group was higher than that in the MMI group $(\mathrm{P}=0.0034)$. However, difference in FT4 between before and 4 days after discontinuation of drugs of the KI group was slightly higher than that of the MMI group, but it was not significant $(\mathrm{P}=0.1557)$. Regarding thyroid function at 1 year after RAIT, the "R" rate of the KI group was slightly higher than that of the MMI group, but it was not significant (P $=0.0932$ ). Thyroid gland weight at 1 year after RAIT of the KI group was lower than that of the MMI group $(\mathrm{P}=0.0065)$. The dose of drugs at 1 year after RAIT was added as supplementary data in Table 2 .

\section{Discussion}

In Japan, RAIT is the second or third choice for GD treatment [2]. However, institutions carrying out RAIT have been gradually increasing. Therefore, when patients show adverse effects of ATDs or cannot go into remission by ATDs, RAIT or thyroidectomy is often chosen. Thyrotoxicosis is one of the significant complications associated with RAIT. Not only transient exacerbation of thyrotoxicosis, but also thyroid storm has been reported [17-19]. To reduce such risks, ATDs are administered in preparation for RAIT in Japan. In addition, in patients who show adverse effects of ATDs or whose hyperthyroidism cannot be controlled by only ATDs, KI is also often administered as a treatment before RAIT. In fact, Zakavi et al reported that a shorter discontinuation of MMI before RAIT did not influence the effects of RAIT and may be preferable in most patients [4]. On the other hand, the influence on RAIT of KI discontinuation before RAIT was seldom reported. Some reports recommended lithium administration as a pretreatment for RAIT [20]. However, administration of lithium for hyperthyroidism is an off-label prescription in Japan. Therefore, it is difficult to routinely administer lithium to GD patients suffering from adverse effects of ATDs. According to the guidelines for RAIT from JTA in 2007, the KI discontinuation 3 - 4 days in prior to RAIT is effective. However, the data and/or literature supporting this were not available in the guidelines [15]. Therefore, in this study, we collected the data of GD patients who were prepared for RAIT with KI and evaluated whether a short duration of KI discontinuation before RAIT is a safe and useful method in GD patients showing adverse ATD effects.

The significantly lower RAIU of the KI group compared with that of the MMI group (Table 2) suggests that a 4-day discontinuation of KI may not be sufficient for adequate RAIU. Hiraiwa et al reported that RAIU of Japanese GD patients without iodine restriction was $42.4 \pm 17.6 \%$ (SD) [21]. In our study, KI was administered at a median dose of $150 \mathrm{mg}$ in the KI group and hyperthyroidism was considerably treated before RAIT. High doses of iodine suppress RAIU and thyroid hormone secretion $[22,23]$. Therefore, RAIU before KI discontinuation may have been suppressed by the above administered dose of iodine, and a 4-day discontinuation of KI contributed to improve RAIU to that equivalent of Japanese GD patients without iodine restriction.

Thyroid gland weight did not show significant difference between KI and MMI group, but that of the KI group was slightly lower than that of the MMI group. Therefore, in the KI group, although the RAIU was lower than that of the MMI group, the dose of 131-I based on thyroid gland weight was equivalent to that of the MMI group. According to Beierwaltes' reports, the dose of 131-I based on thyroid gland weight in both groups was adequate to treat hyperthyroidism [16]. Thyroid function tests at 1 year after RAIT did not show significant statistical difference between the KI and MMI groups. However, the " $\mathrm{R}$ " rate of the KI group was slightly higher than that of the MMI group. The result associated with thyroid gland weight at 1 year after RAIT was also consistent with this result. There were many patients who could not go into remission with ATDs in the MMI group; however, almost all 
patients showed adverse effects from ATDs in the KI group. The differences in aggressiveness and/or resistance to ATDs between the two groups may have contributed to the difference in the "R" rate. Therefore, it may be difficult to demonstrate the efficiency of preparation for RAIT by KI with MMI group comparison. However, our results demonstrated that GD patients without large goiter and/or highly aggressive disease could achieve a good outcome with KI preparation for RAIT.

Regarding thyroid function after a 4-day discontinuation of KI, FT4 in the KI group was higher than that in the MMI group. It is well known that the effects of KI in GD often diminish during administration, described as the "escape phenomenon" [24]. However, Okamura et al reported that many GD patients who were treated with KI continuously for a long duration went into remission in Japan [14]. We continued KI treatment for GD patients unless they had significant hyperthyroidism and almost all patients in the KI group showed mild hyperthyroxinemia. This background may explain the higher FT4 at RAIT in the KI group than that in the MMI group. However, the difference in FT4 between before and after drug discontinuation was not significant between the two groups, suggesting that the influence of the 4-day discontinuation of KI on aggravation of thyroid function was equivalent to that of the 4-day discontinuation of MMI.

\section{Conclusion}

In the present study, our results suggested that preparation for RAIT by KI predicted a good RAIT outcome, and that a 4-day discontinuation of KI before RAIT is a useful and safe procedure to avoid RAIT-associated thyroid function deterioration. In conclusion, RAIT can be performed safely by discontinuing KI 4 days before the treatment and can achieve a good outcome in GD patients without large goiter and/or highly aggressive disease.

\section{Acknowledgments}

We gratefully acknowledge the work of clinical staff of Yamashita Thyroid and Parathyroid Clinic.

\section{References}

1. Becker DV, Sawin CT. Radioiodine and thyroid disease: the beginning. Semin Nucl Med. 1996;26(3):155-164.

2. Wartofsky L, Glinoer D, Solomon B, Nagataki S, Lagasse R, Nagayama Y, Izumi M. Differences and similarities in the diagnosis and treatment of Graves' disease in Europe, Japan, and the United States. Thyroid. 1991;1(2):129135.

3. Burch HB, Burman KD, Cooper DS. A 2011 survey of clinical practice patterns in the management of Graves' disease. J Clin Endocrinol Metab. 2012;97(12):45494558.

4. Zakavi SR, Khazaei G, Sadeghi R, Ayati N, Davachi B, Bonakdaran S, Jabbari Nooghabi M, et al. Methimazole discontinuation before radioiodine therapy in patients with Graves' disease. Nucl Med Commun. 2015;36(12):12021207.

5. Wartofsky L. Low remission after therapy for Graves disease. Possible relation of dietary iodine with antithyroid therapy results. JAMA. 1973;226(9):1083-1088.

6. Solomon BL, Evaul JE, Burman KD, Wartofsky L. Remission rates with antithyroid drug therapy: continuing influence of iodine intake? Ann Intern Med. 1987;107(4):510512.

7. Azizi F. Environmental iodine intake affects the response to methimazole in patients with diffuse toxic goiter. J Clin Endocrinol Metab. 1985;61(2):374-377.

8. Benker G, Vitti P, Kahaly G, Raue F, Tegler L, Hirche $\mathrm{H}$, Reinwein D. Response to methimazole in Graves' disease. The European Multicenter Study Group. Clin Endocrinol (Oxf). 1995;43(3):257-263.

9. Alexander WD, Harden RM, Koutras DA, Wayne E. Influence of iodine intake after treatment with antithyroid drugs. Lancet. 1965;2(7418):866-868.

10. Laurberg P. Iodine intake - what are we aiming at? J Clin Endocrinol Metab. 1994;79(1):17-19.

11. David SC. Treatment of thyrotoxicosis. In: Lewis EB, David SC, eds. Werner \& Ingbar's The Thyroid. A Fundamental and Clinical Text. 10th ed. Philadelphia: Lippincott Williams \& Wilkins; 2013. p. 492-516.

12. Hiraiwa T, Ito M, Imagawa A, Takamatsu J, Kuma K, Miyauchi A, Hanafusa T. Restriction of dietary Iodine does not ameliorate the early effect of anti-thyroid drug therapy for Graves' disease in an area of excessive iodine intake. J Endocrinol Invest. 2006;29(4):380-384.

13. Takata K, Amino N, Kubota S, Sasaki I, Nishihara E, Kudo T, Ito M, et al. Benefit of short-term iodide supplementation to antithyroid drug treatment of thyrotoxicosis due to Graves' disease. Clin Endocrinol (Oxf). 2010;72(6):845-850.

14. Okamura K, Sato K, Fujikawa M, Bandai S, Ikenoue H, Kitazono T. Remission after potassium iodide therapy in patients with Graves' hyperthyroidism exhibiting thionamide-associated side effects. J Clin Endocrinol Metab. 2014;99(11):3995-4002.

15. Konishi J. Preparations before radioacitive iodine therapy for Graves' disease. In: Nakamura H, Konishi J, eds. Guideline for the Treatment of Graves' disease in Japan (in Japanese). Tokyo: Nankodo; 2011. p. 188-193.

16. Beierwaltes WH. The treatment of hyperthyroidism with iodine-131. Semin Nucl Med. 1978;8(1):95-103.

17. Tamagna EI, Levine GA, Hershman JM. Thyroid-hormone concentrations after radioiodine therapy for hyperthyroidism. J Nucl Med. 1979;20(5):387-391.

18. McDermott MT, Kidd GS, Dodson LE, Jr., Hofeldt FD. Radioiodine-induced thyroid storm. Case report and literature review. Am J Med. 1983;75(2):353-359.

19. Kadmon PM, Noto RB, Boney CM, Goodwin G, Gruppuso PA. Thyroid storm in a child following radioactive iodine (RAI) therapy: a consequence of RAI versus withdrawal of antithyroid medication. J Clin Endocrinol Metab. 2001;86(5):1865-1867.

20. Bogazzi F, Giovannetti C, Fessehatsion R, Tanda ML, 
Campomori A, Compri E, Rossi G, et al. Impact of lithium on efficacy of radioactive iodine therapy for Graves' disease: a cohort study on cure rate, time to cure, and frequency of increased serum thyroxine after antithyroid drug withdrawal. J Clin Endocrinol Metab. 2010;95(1):201-208.

21. Hiraiwa $T$, Ito $M$, Imagawa A, Isotani $H$, Takamatsu J, Kuma K, Miyauchi A, et al. High diagnostic value of a radioiodine uptake test with and without iodine restriction in Graves' disease and silent thyroiditis. Thyroid. 2004;14(7):531-535.
22. Nagataki S, Shizume K, Nakao K. Effect of iodide on thyroidal iodine turnover in hyperthyroid subjects. J Clin Endocrinol Metab. 1970;30(4):469-478.

23. Wartofsky L, Ransil BJ, Ingbar SH. Inhibition by iodine of the release of thyroxine from the thyroid glands of patients with thyrotoxicosis. J Clin Invest. 1970;49(1):7886.

24. Emerson $\mathrm{CH}$, Anderson AJ, Howard WJ, Utiger RD. Serum thyroxine and triiodothyronine concentrations during iodide treatment of hyperthyroidism. J Clin Endocrinol Metab. 1975;40(1):33-36. 